
\title{
28 Research Suare \\ Experimental evaluation of thermodynamic cost and speed limit in living cells via information geometry
}

\section{Keita Ashida}

The University of Tokyo

\section{Kazuhiro Aoki}

National Institutes of Natural Sciences, National Institute for Basic Biology https://orcid.org/00000001-7263-1555

\section{Sosuke Ito ( $\nabla$ sosuke.ito@ubi.s.u-tokyo.ac.jp )}

The University of Tokyo https://orcid.org/0000-0001-6954-1321

\section{Physical Sciences - Article}

Keywords: Chemical Reaction Information Processing, Stochastic Thermodynamics, Informationgeometric Method, Thermodynamic Uncertainty Relations, Signal Transduction Pathways

Posted Date: December 9th, 2020

DOI: https://doi.org/10.21203/rs.3.rs-118501/v1

License: (1) (1) This work is licensed under a Creative Commons Attribution 4.0 International License. Read Full License 


\title{
Experimental evaluation of thermodynamic cost and speed limit in living cells via information geometry
}

\author{
Keita Ashida, ${ }^{1}$ Kazuhiro Aoki, ${ }^{2,3,4}$ and Sosuke Ito $^{1,5,6}$ \\ ${ }^{1}$ Universal Biology Institute, The University of Tokyo, 7-3-1 Hongo, Bunkyo-ku, Tokyo 113-0033, Japan \\ ${ }^{2}$ Quantitative Biology Research Group, Exploratory Research Center on Life and Living Systems (ExCELLS), \\ National Institutes of Natural Sciences, 5-1 Higashiyama, Myodaiji-cho, Okazaki, Aichi 444-8787, Japan \\ ${ }^{3}$ Division of Quantitative Biolny, National Institute for Basic Biology, \\ National Institutes of Natural Sciences, 5-1 Higashiyama, Myodaiji-cho, Okazaki, Aichi 444-8787, Japan \\ ${ }^{4}$ Department of Basic Biology, School of Life Science, \\ SOKENDAI (The Graduate University for Advanced Studies), \\ 5-1 Higashiyama, Myodaiji-cho, Okazaki, Aichi 444-8787, Japan \\ ${ }^{5}$ Department of Physics, The University of Tokyo, 7-3-1 Hongo, Bunkyo-ku, Tokyo 113-0033, Japan \\ ${ }^{6}$ JST, PRESTO, 4-1-8 Honcho, Kawaguchi, Saitama, 332-0012, Japan
}

Chemical reactions are responsible for information pro- 56 cessing in living cells, and their accuracy and speed have 57 been discussed from a thermodynamic viewpoint [1-5]. 58 The recent development in stochastic thermodynamics en- 59 ables evaluating the thermodynamic cost of information 60 processing [6-8]. However, because experimental estima- 61 tion of the thermodynamic cost based on stochastic ther- 62 modynamics requires a sufficient number of samples [9], 63 it is only estimated in simple living systems, such as RNA 64 folding [10] and $\mathbf{F}_{1}$-ATPase [11]. Therefore, it is chal- 65 lenging to estimate the thermodynamic cost of informa- 66 tion processing by chemical reactions in living cells. Here, 67 we evaluated the thermodynamic cost and its efficiency 68 of information processing in living systems at the single- 69 cell level for the first time by establishing an information- 70 geometric method to estimate them with a relatively small 71 number of samples. We evaluated the thermodynamic cost 72 of the extracellular signal-regulated kinase (ERK) phos- 73 phorylation from the time series of the fluorescence imag- 74 ing data by calculating the intrinsic speed in information 75 geometry. We also evaluated a thermodynamic efficiency 76 based on the thermodynamic speed limit $[8,12,13]$, and thus this paper reports the first experimental test of ther- ${ }^{77}$ modynamic uncertainty relations in living systems. Our ${ }^{78}$ evaluation revealed the change of the efficiency under the ${ }^{79}$ conditions of different cell densities and its robustness to ${ }^{80}$ the upstream pathway perturbation. Because our ap- 81 proach is widely applicable to other signal transduction ${ }^{82}$ pathways, such as the G-protein coupled receptor path- ${ }^{83}$ ways for sensation [14], it would clarify efficient mecha- ${ }^{84}$ nisms of information processing in such a living system.

The Gibbs free energy change mainly drives information 87 transmission in living cells, and its mechanism follows ther- 88 modynamic laws. For example, systems biology reveals a 89 deep connection between information transmission accuracy 90 and the Gibbs free energy change in a cell [I, 2]. In recent 91 developments of stochastic thermodynamics [15] and chem- 92 ical thermodynamics [16, 17], a similar connection occurs 93 more deeply. The trade-off relations among speed, thermo- 94 dynamic cost, and accuracy in a living cell have been pro- 95 posed [3, 4], and its general thermodynamic bound of infor- 96 mation processing in signal transduction has been discussed 97 in terms of Maxwell's demon [5, 18-20]. Moreover, as thermodynamic generalizations of uncertainty relations, thermodynamic trade-off relations have been intensively studied in terms of thermodynamic uncertainty relations [21, 22] and thermodynamic speed limits [8, 23-29]. These trade-off relations are mainly based on mathematical properties of the Fisher information [8, 12, 30], which gives a metric of information geometry [31]. Historically, information geometry has been considered as a possible choice of differential geometry for equilibrium thermodynamics [7, 32]. In recent years, information geometry meets nonequilibrium thermodynamics such as stochastic thermodynamics [8, 12, 33] and chemical thermodynamics [13], and it provides a unified framework to derive these trade-off relations in living systems [ [8, 12, 13]]. While theoretical progress of thermodynamic trade-off relations has been intensively made for biological applications, the thermodynamic uncertainty relation has been experimentally tested only in artificial systems [34, 35]. An experimental test of thermodynamic trade-off relations in living systems has not been reported, and the thermodynamic speed limit has not been tested even in artificial systems.

This paper experimentally evaluated the thermodynamic cost by quantifying the Fisher information of time from the fluorescence imaging of the ERK phosphorylation in normal rat kidney epithelial (NRK-52E) cells. In information geometry, the Fisher information of time is regarded as the square of the intrinsic speed. This Fisher information is calculated from the time evolution of the phosphorylated ERK fraction, which can be experimentally measured by the Förster resonance energy transfer (FRET) signal. We also evaluated other information-geometric quantities for activation and inactivation processes from this Fisher information, and these information-geometric quantities illustrate the thermodynamic cost of the ERK phosphorylation, which is related to the Gibbs free energy change and the fluctuation-response ratio. We focused on the thermodynamic speed limit based on these information-geometric quantities and found that the efficiency ranges from 0.4 to 0.9 for the ERK phosphorylation in living NRK-52E cells. We quantified the thermodynamic variability for cell density changes and the perturbation of the upstream Raf pathway. While the cell density increases the thermodynamic cost and reduces efficiency, the Raf inhibitor 
addition reduces the thermodynamic cost, but the efficiency is 153 robust to the Raf inhibitor addition.

The information-geometric method proposed in this paper ${ }_{155}$ is generally applicable to switching dynamics between the ac-156 tive state and the inactive state. We consider the following ${ }_{157}$ chemical reaction

$$
A_{\mathrm{off}} \underset{k_{\mathrm{off}}}{\stackrel{k_{\mathrm{on}}\left(s^{\prime}\right)}{\rightleftarrows}} A_{\mathrm{on}}
$$

where $A_{\text {on }}$ is an active state, $A_{\text {off }}$ is an inactive state, $s^{\prime}$ is ${ }^{158}$ stimulus, and $k_{\mathrm{on}}\left(s^{\prime}\right)$ and $k_{\mathrm{off}}$ are rate constants. Because ${ }^{159}$ $k_{\text {on }}\left(s^{\prime}\right)$ can depend on stimulus $s^{\prime}$, it causes a pulsatile re- ${ }^{160}$ sponse of $A_{\mathrm{on}}$ if this stimulus $s^{\prime}$ is excitable. This paper ${ }^{161}$ focuses on the ERK phosphorylation in the Ras-Raf-MEK- ${ }^{162}$ ERK pathway that shows a pulsatile response [36-38]. $\operatorname{In}^{163}$ Fig. Ta, we show the Ras-Raf-MEK-ERK pathway that relays ${ }_{165}^{164}$ extracellular stimuli such as growth factors from the plasma ${ }^{165}$ membrane to targets in the cytoplasm and nucleus. This three-tiered Raf-MEK-ERK mitogen-activated protein kinase ${ }^{167}$ (MAPK) cascade plays an essential role in various cellular ${ }_{169}^{168}$ processes, including cell proliferation, differentiation, and tu- ${ }^{169}$ morigenesis [39]. Upon growth factor stimulation, the re- ${ }^{-170}$ ceptor tyrosine kinase (RTK) activates the Ras small GTPase at the plasma membrane, which recruits and activates the ${ }^{172}$ Raf. The activated Raf induces activation and phosphoryla- ${ }^{173}$ tion of the MEK. The upstream kinase MEK phosphorylates ${ }^{174}$ the ERK to increase kinase activation of the ERK. The phosphorylated ERK is finally dephosphorylated by phosphatases, thereby shutting down the ERK activation. The phosphorylated MEK catalyzes a phosphate transfer from the adenosine ${ }^{175}$ triphosphate (ATP) to the ERK, and the Gibbs free energy ${ }^{176}$ difference of ATP hydrolysis thermodynamically drives this ${ }^{177}$ phosphorylation of the ERK [39, 40]. Here, $A_{\text {on }}$ corresponds ${ }^{178}$ to a phosphorylated state of the ERK, $A_{\text {off }}$ corresponds to a ${ }^{179}$ nonphosphorylated state of the ERK, and $s^{\prime}$ corresponds to ${ }^{180}$ the stimulus by upstream proteins in the Ras-Raf-MEK-ERK ${ }^{181}$ pathway, respectively. We try to estimate the thermodynamic ${ }^{182}$ cost of the ERK phosphorylation in living cells using the time ${ }^{183}$ series of the phosphorylated ERK fraction, which can be ex-184 perimentally measured by the FRET technique at the single-185 cell level [37].

We discuss the thermodynamic cost estimation from the ${ }^{187}$ time series of the phosphorylated ERK fraction $P_{1}={ }^{188}$ $\left[A_{\text {on }}\right] /\left(\left[A_{\text {on }}\right]+\left[A_{\text {off }}\right]\right)$, where $\left[A_{\text {on }}\right]$ and $\left[A_{\text {off }}\right]$ are concen-189 trations corresponding to $A_{\text {on }}$ and $A_{\text {off }}$, respectively (see also ${ }^{190}$ Supplementary Note). In general, it is hard to estimate the Gibbs free energy change in living cells because it needs prior knowledge about the equilibrium concentration [16]. However, this equilibrium concentration is not estimated well ${ }_{191}$ from the time series of $P_{1}$. We propose a novel informationgeometric method to estimate the thermodynamic cost from $P_{1}$, which does not require prior knowledge about the equilibrium concentration. Because the total concentration $\left[A_{\text {tot }}\right]=$ $\left[A_{\text {off }}\right]+\left[A_{\text {on }}\right]$ is conserved, the nonphosphorylated and phosphorylated ERK fractions $P_{0}=1-P_{1}=\left[A_{\text {off }}\right] /\left[A_{\text {tot }}\right]$ and $_{192}$ $P_{1}=\left[A_{\text {on }}\right] /\left[A_{\text {tot }}\right]$ can be regarded as the probability distribution, and a Riemannian manifold can be introduced as the set of probability distributions in information geometry [3T].
This method focuses on an intrinsic speed $d s / d t=\sqrt{d s^{2} / d t^{2}}$ on this manifold related to the thermodynamic cost, such as the Gibbs free energy change and the fluctuation-response ratio of the ERK phosphorylation [ [8, [2, [3] ]. The square of the intrinsic speed is given by the Fisher information of time

$$
\frac{d s^{2}}{d t^{2}}=\sum_{i=0}^{1} P_{i}\left(\frac{d \ln P_{i}}{d t}\right)^{2}=\sum_{i=0}^{1} \frac{1}{P_{i}}\left(\frac{d P_{i}}{d t}\right)^{2} .
$$

Because $d s^{2} / d t^{2}$ only consists of the concentration fraction $P_{i}$ and its change speed $d P_{i} / d t$, we can estimate it from the time series of $P_{1}$.

The square of the intrinsic speed can quantify the thermodynamic cost of ERK phosphorylation (see also Supplementary Note). Under near-equilibrium condition, we obtain $d s^{2} / d t^{2} \simeq-(d \sigma / d t) /\left(2 R\left[A_{\text {tot }}\right]\right)$, where $R$ is the gas constant, and $\sigma$ is the entropy production that is the minus sign of the Gibbs free energy change over the temperature [13]]. Then, the Fisher information of time is proportional to the entropy production rate change under near-equilibrium condition. Even for a system far from equilibrium, the square of the intrinsic speed gives the fluctuation-response ratio of the ERK phosphorylation. Let $f_{i}$ be an observable of the ERK states, where $i=0(i=1)$ means the nonphosphorylated (phosphorylated) state. The square of the intrinsic speed $d s^{2} / d t^{2}$ is equal to the fluctuation-response ratio

$$
\frac{d s^{2}}{d t^{2}}=\frac{\left|d_{t}\langle f\rangle_{t}\right|^{2}}{\operatorname{Var}[f]_{t}},
$$

where $\langle f\rangle_{t}=\sum_{i} P_{i}(t) f_{i}$ is the mean value, $\left|d_{t}\langle f\rangle_{t}\right|=$ $\left|d\langle f\rangle_{t} / d t\right|$ implies the time-response of the observable, and $\operatorname{Var}[f]_{t}=\left\langle f^{2}\right\rangle_{t}-\langle f\rangle_{t}^{2}$ implies the observable fluctuation. This relation is a consequence of the Cramér-Rao inequality for an efficient estimator, where any observable of a binary state can be regarded as an efficient estimator.

As shown in Fig. Wb, a pulsatile response consists of activation and inactivation processes, and we introduce informationgeometric quantities of these processes as measures of the thermodynamic cost and the speed limit's efficiency (see also Supplementary Note). During the activation (inactivation) process, the phosphorylated ERK fraction $P_{1}$ is monotonically increasing (decreasing) in time. The activation (inactivation) starts at time $t_{0}=t_{\text {ini }}\left(t_{0}=t_{\text {peak }}\right)$ and ends at time $t_{1}=t_{\text {peak }}\left(t_{1}=t_{\text {fin }}\right)$. We here introduce three informationgeometric quantities [ [, 8$]$ ] , the action

$$
\mathcal{C}=\frac{1}{2} \int_{t_{0}}^{t_{1}} d t \frac{d s^{2}}{d t^{2}}
$$

the length

$$
\mathcal{L}=\int_{t_{0}}^{t_{1}} \sqrt{\frac{d s^{2}}{d t^{2}}} d t=2 \arccos \left[\sum_{i=0}^{1} \sqrt{\left.\left.P_{i}\right|_{t=t_{0}} P_{i}\right|_{t=t_{1}}}\right],
$$

and the mean velocity

$$
\mathcal{V}=\frac{\mathcal{L}}{t_{1}-t_{0}}
$$


a

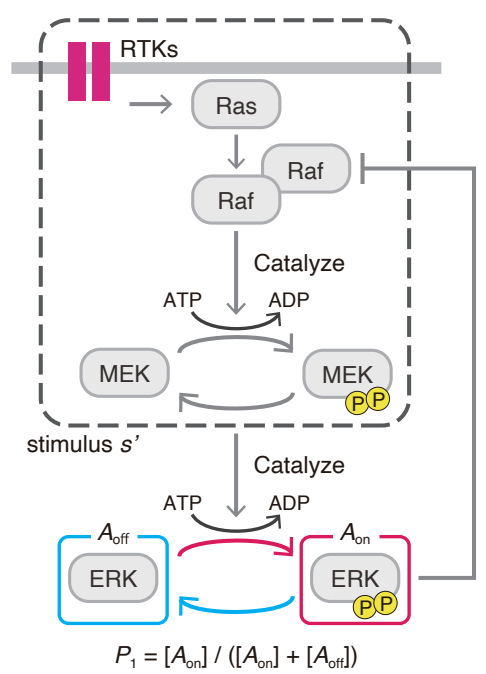

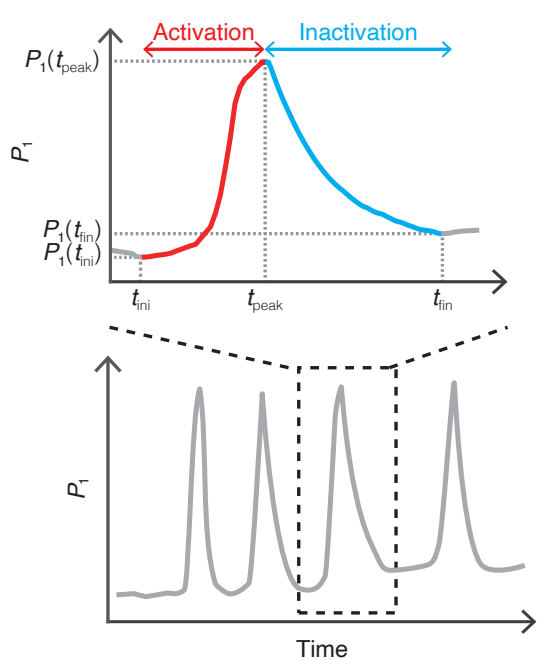

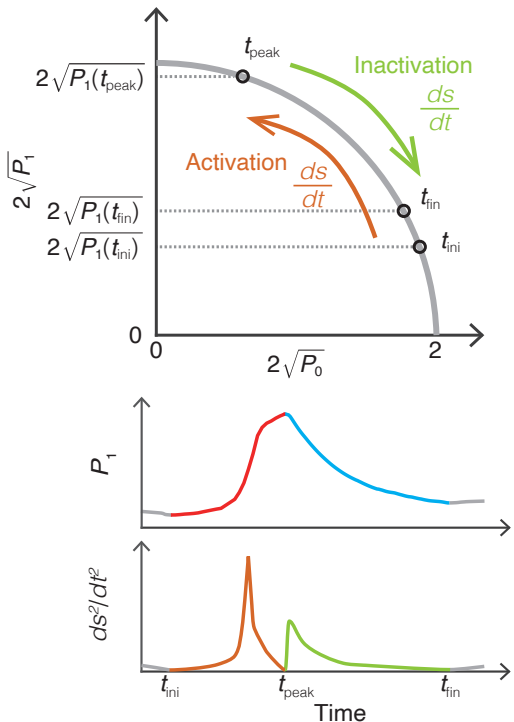

Fig. 1: Schematic of the ERK phosphorylation, the time series of the phosphorylated ERK fraction $P_{1}$, and the square of the intrinsic speed $d s^{2} / d t^{2}$. a The Raf-MEK-ERK MAP pathway as an example of switching dynamics between the active state $A_{\text {on }}$ and the inactive state $A_{\text {off }}$. The phosphorylated and nonphosphorylated ERKs correspond to $A_{\text {on }}$ and $A_{\text {off }}$, respectively. The upstream proteins correspond to the stimulus $s^{\prime}$, and the Raf on the upstream pathway affects the phosphorylated ERK. $\mathbf{b}$

Typical behavior of the phosphorylated ERK fraction $P_{1}$ in the activation and inactivation processes. The time $t_{\text {ini }}\left(t_{\text {peak }}\right)$ indicates the beginning of the activation (inactivation), and the time $t_{\text {peak }}\left(t_{\text {fin }}\right)$ indicates the end of the activation (inactivation).

c Schematic of the manifold in information geometry and the intrinsic speed $d s / d t$ on this manifold. The activation and inactivation processes give at least two peaks in the time series of $d s^{2} / d t^{2}$, because $d s^{2} / d t^{2}=0$ at times $t_{\text {ini }}, t_{\text {peak }}$, and $t_{\text {fin }}$.

during the process from time $t_{0}$ to $t_{1}$. The action $\mathcal{C}$ quan-215 tifies the thermodynamic cost of the process because the ac-216 tion $\left.\mathcal{C} \simeq \sigma\right|_{t=t_{0}} /\left(4 R\left[A_{\text {tot }}\right]\right)$ is approximately proportional to 217 the entropy production rate at time $t_{0}$ under near-equilibrium 218 condition. Even for a system far from equilibrium, the action 219 $\mathcal{C}$ can be interpreted as the thermodynamic cost in terms of the fluctuation-response ratio in Eq. (B) and becomes large if ${ }_{221}^{220}$ the observable change speed $\left|d_{t}\langle f\rangle_{t}\right|$ is relatively larger than ${ }^{221}$ its fluctuation $\sqrt{\operatorname{Var}[f]_{t}}$ during the process. The length $\mathcal{L}$ is $_{223}$ given by twice the Bhattacharyya angle, which is a measure ${ }_{224}$ of a difference between two concentration fractions at time $t_{0_{225}}$ and time $t_{1}$. The mean velocity $\mathcal{V}$ quantifies the speed of the ${ }_{226}$ concentration fraction change during the process. In informa- ${ }_{227}$ tion geometry, $\mathcal{L}$ is regarded as the arc length of a circle in ${ }_{228}$ $2 \sqrt{P_{i}}$ coordinate, and $d s / d t$ is the intrinsic speed on this cir- ${ }_{229}$ cle (see also Fig. Пc). From the Cauchy-Schwarz inequality ${ }_{230}$ we obtain the thermodynamic speed limit [8]

$$
\mathcal{L}^{2} \leq 2 \mathcal{C}\left(t_{1}-t_{0}\right)
$$

which is a trade-off relation between the thermodynamic $\operatorname{cost}^{234}$ $\mathcal{C}$ and the transition time $t_{1}-t_{0}$ during the process. To quan- ${ }^{235}$ tify how much the thermodynamic cost converts into the con- ${ }^{236}$ centration fraction change speed, we can consider the speed 237 limit's efficiency [8]

$$
\eta=\frac{\mathcal{L}^{2}}{2\left(t_{1}-t_{0}\right) \mathcal{C}}=\frac{\mathcal{V} \mathcal{L}}{2 \mathcal{C}} .
$$

The efficiency $\eta$ satisfies $0 \leq \eta \leq 1$, and $\eta=1(\eta=0)$ implies that this conversion is most efficient (inefficient). The efficiency becomes higher if the intrinsic speed is close to constant because $\eta=1$ if and only if the intrinsic speed $d s / d t$ is constant regardless of time, $(d / d t)\left|d s^{2} / d t^{2}\right|=0$.

We experimentally measured the phosphorylated ERK fraction in living NRK-52E cells with the FRET-based ERK biosensor, the EKAREV-NLS (Fig. Za) [41]]. By comparing the fluorescence ratio of cells with the phosphorylated ERK fraction obtained from the western blotting (Fig. Zlb), we quantified $P_{1}$ and the square of the intrinsic speed $d s^{2} / d t^{2}$ for the ERK activation ( $p=a)$ and inactivation $(p=i)$ processes under the condition of different cell densities: $2.0 \times 10^{3}$ cells $/ \mathrm{cm}^{2}$ (low, $\left.d=\mathrm{L}\right), 2.0 \times 10^{4}$ cells $/ \mathrm{cm}^{2}$ (medium, $\left.d=\mathrm{M}\right)$, and $2.0 \times 10^{5}$ cells $/ \mathrm{cm}^{2}$ (high, $d=\mathrm{H}$ ), where the indices $p \in\{\mathrm{a}, \mathrm{i}\}$ and $d \in\{\mathrm{L}, \mathrm{M}, \mathrm{H}\}$ regard the process and cell density, respectively. The pulses of the ERK activation were observed under these conditions (Fig. Dاc and Supplementary Video 1), and the behavior of $d s^{2} / d t^{2}$ characterizes these conditions $p$ and $d$ (Fig. Dاd). It reveals thermodynamic differences of the intracellular ERK activation under these conditions.

The information-geometric quantities $\{\mathcal{C}, \mathcal{L}, \mathcal{V}\}_{d}^{p}$ also differentiate these conditions (Fig. [3] and Extended Data Table 2). Firstly, we discuss the histogram of the action. The mean value of the action $\overline{\mathcal{C}_{d}^{p}}$ becomes larger as cell density increases $\overline{\mathcal{C}_{\mathrm{H}}^{p}}>\overline{\mathcal{C}_{\mathrm{M}}^{p}}>\overline{\mathcal{C}_{\mathrm{L}}^{p}}$ for both $p=\mathrm{a}$ and $p=\mathrm{i}$. It reflects the 
a

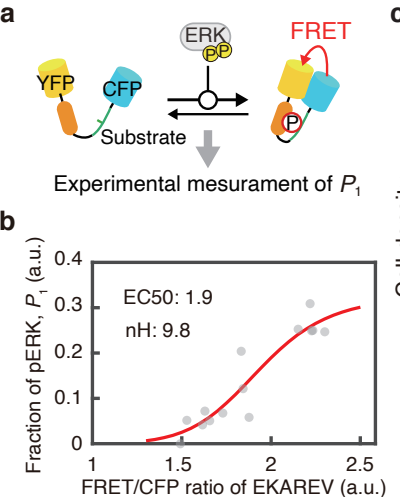

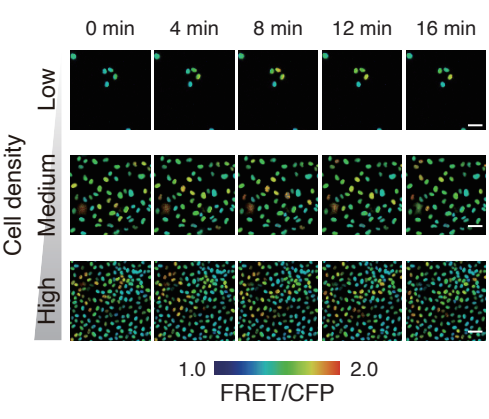

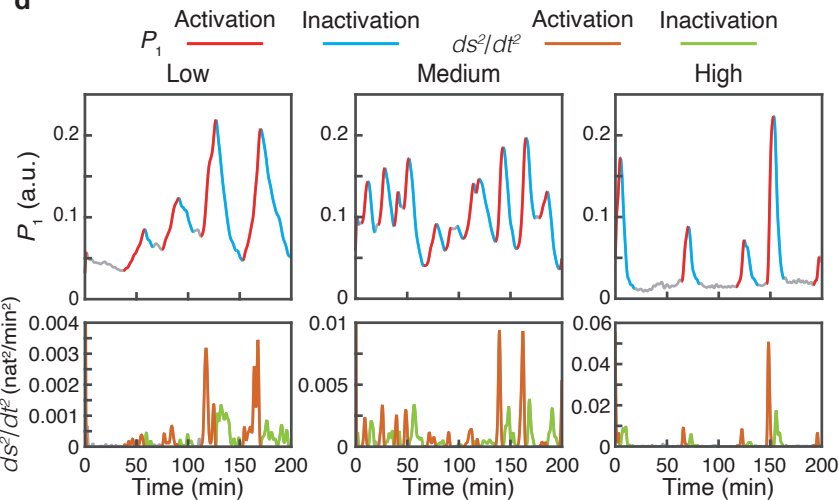

Fig. 2: The dynamics of the ERK phosphorylation under the conditions of different cell densities. a Schematic of the

FRET-based ERK sensor. The phosphorylated ERK fraction $P_{1}$ can be measured from this FRET signal. b The relation between the FRET/CFP ratios of the EKAREV-NLS and the phosphorylated ERK fraction $P_{1}$. The red line indicates the fitted curve of the Hill equation. c Representative images of the phosphorylated ERK under the conditions of low, medium, and high cell densities. Scale bars indicate $50 \mu \mathrm{m}$. d Representative examples of the ERK phosphorylation dynamics under low (left), medium (middle), and high (right) cell densities. The unit (a.u.) indicates the arbitrary unit, and the unit (nat) indicates the natural unit of information.
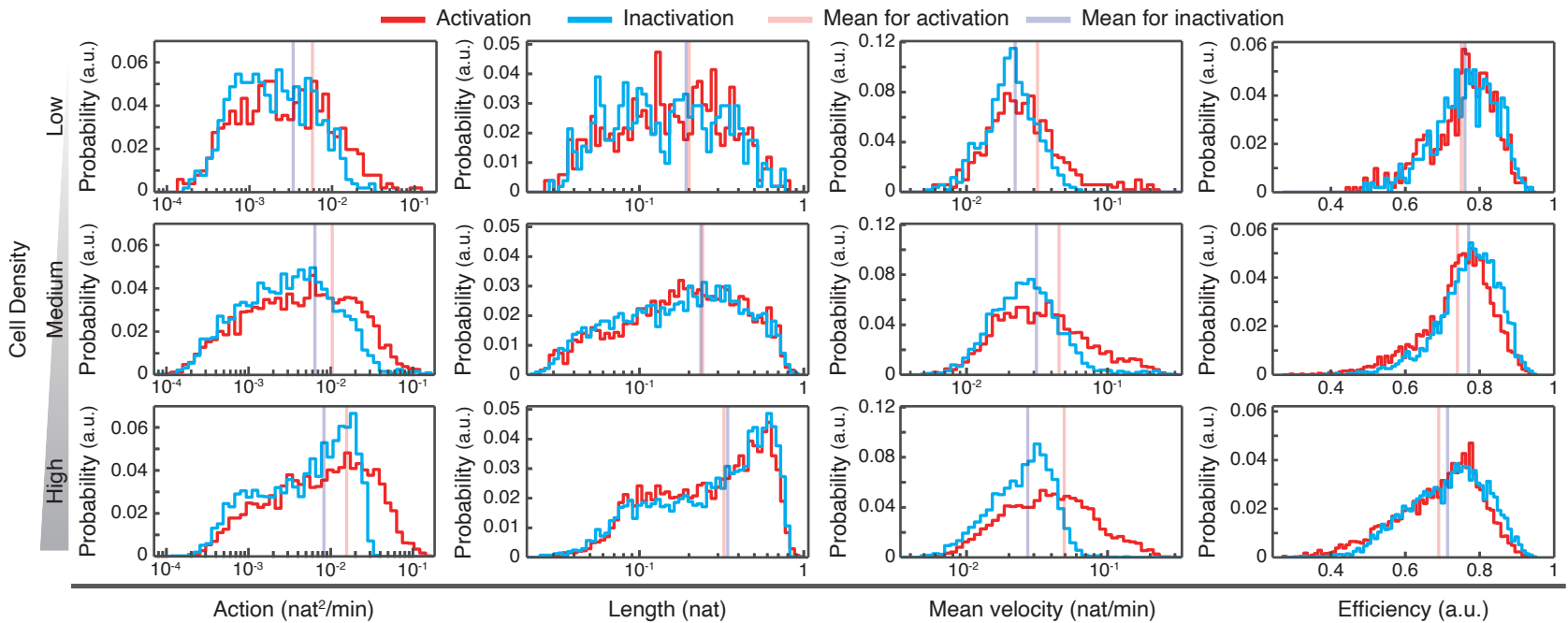

Fig. 3: The histograms of the action $\mathcal{C}$, length $\mathcal{L}$, mean velocity $\mathcal{V}$, and efficiency $\eta$ under the conditions of different cell densities. The sample size, mean values, variances, and results of statistical tests are listed in Extended Data Table 1, 2.

fact that the speed under the condition of the high density ap-253 peared faster than the low density. The difference of $\overline{\mathcal{C}_{d}^{p}}$ be-254 tween the high (medium) and low densities is at least twice 255 $\left(\overline{\mathcal{C}_{\mathrm{H}}^{\mathrm{a}}} / \overline{\mathcal{C}_{\mathrm{L}}^{\mathrm{a}}} \simeq 2.7, \overline{\mathcal{C}_{\mathrm{H}}^{\mathrm{i}}} / \overline{\mathcal{C}_{\mathrm{L}}^{\mathrm{i}}} \simeq 2.4\right)$. In comparison with the activa-256 tion and inactivation processes, $\overline{\mathcal{C}_{d}^{\mathrm{a}}}$ is approximately twice $\mathrm{as}^{257}$ much as $\overline{\mathcal{C}_{d}^{\mathrm{i}}}$, and the distributions for the activation have longer ${ }^{258}$ tails than the inactivation. Because the action is a measure of ${ }^{259}$ the thermodynamic cost, these results suggest that the activa- ${ }^{260}$ tion's thermodynamic cost is larger than the inactivation's one and becomes larger as cell density increases. Secondly, we ${ }^{261}$ discuss the histogram of the length. While the length does not ${ }_{263}^{262}$ distinguish the activation process from the inactivation process, the mean value of the length $\overline{\mathcal{L}_{d}^{p}}$ differentiates the cell densities. It reflects the fact that the peak of the spike becomes higher as the cell density increases. Finally, we discuss the histogram of the mean velocity. The mean value of the mean velocity for the activation $\overline{\mathcal{V}}_{d}^{\mathrm{a}}$ is larger than the inactivation $\overline{\mathcal{V}_{d}^{\mathrm{i}}}$. It shows that the speed of the activation is faster than the inactivation.

From these information-geometric quantities $\{\mathcal{C}, \mathcal{L}, \mathcal{V}\}_{d}^{p}$, we obtained the speed limit's efficiency $\eta_{d}^{p}=\mathcal{V}_{d}^{p} \mathcal{L}_{d}^{p} /\left(2 \mathcal{C}_{d}^{p}\right)$ (Fig. [3 right column). The mean value of the speed limit's 
efficiency is almost $\overline{\eta_{d}^{p}} \simeq 0.7$ under these conditions, and the $e_{313}$ distribution of $\eta_{\mathrm{H}}^{p}$ shifted to lower than those of $\eta_{\mathrm{L}}^{p}$ and $\eta_{\mathrm{M}^{314}}^{p}$ (see also Extended Data Table 2). The efficiencies range be-315 tween $\eta_{d}^{p}=0.4$ and $\eta_{d}^{p}=0.9$, and the distributions' shapes 316 are biased to the higher efficiency. We can detect that the ac- ${ }_{317}$ tivation process is less efficient than the inactivation process $\eta_{d}^{\mathrm{a}}<\eta_{d}^{\mathrm{i}}$. We also find that the efficiency becomes worse in ${ }_{319}$ the higher cell density $\eta_{\mathrm{H}}^{p}<\eta_{\mathrm{L}}^{p}$. These results indicate that ${ }^{320}$ the process is accelerated under the higher cell density, and ${ }_{321}^{320}$ the activation process is also more accelerated than the inactivation process by the thermodynamic cost. This idea is supported by the histogram of $\left|d / d t\left(d s^{2} / d t^{2}\right)\right|$ (Extended Data ${ }^{323}$ Fig. 1), which becomes zero when $\eta=1$. The mean values ${ }^{324}$ of $\left|d / d t\left(d s^{2} / d t^{2}\right)\right|$ for the activation and the high cell density ${ }^{325}$ are larger than that for the inactivation and the low cell den- ${ }^{326}$ sity, respectively (Extended Data Table. 2). The scatter plot ${ }^{327}$ (Extended Data Fig. 2, see also Extended Data Table 4) sug- ${ }^{328}$ gests no correlation between $\eta_{d}^{p}$ and $\{\mathcal{C}, \mathcal{V}\}_{d}^{p}$, while $\mathcal{V}$ and $\mathcal{C}^{329}$ seem to have a correlation. Because cell density modulates the ${ }^{330}$ ERK activation's excitability through both changes in basal ${ }^{331}$ and peak levels of the ERK phosphorylation [37], the cellular ${ }^{332}$ density could affect the efficiency of the pulsatile phosphory- ${ }^{333}$ lation and the thermodynamic cost.

We confirm that these information-geometric quantities 335 show the thermodynamic properties of the ERK phospho-s36 rylation by comparing the Raf inhibitor addition situation.337 The pulsatile dynamics of the ERK activation is generated by stochastic noise from the Raf and feedback loops [37, 39], and ${ }_{339}$ the Gibbs free energy difference of the ERK phosphorylation ${ }_{340}$ is induced by stimulus $s^{\prime}$ from the upstream pathway, includ-341 ing the Raf [39, 40]. Thus, the Raf inhibitor addition reduces 342 the Gibbs free energy change of the ERK phosphorylation and ${ }_{343}$ these information-geometric quantities. To ensure these rela-344 tionships, we measured the ERK phosphorylation dynamics345 under the Raf inhibitor (SB590885) addition and compared $\mathrm{d}_{346}$ its dynamics with the original dynamics before adding the $\mathrm{Raf}_{347}$ inhibitor (Fig. (4). Of note, it is well-known that a low dose of $\mathrm{f}_{348}$ the Raf inhibitor could paradoxically activate the ERK signal-349 ing through the Raf dimerization [42-44]]. The condition of 350 the cell density is medium in this experiment. The applica-351 tion of a low dose (100 $\mathrm{nM})$ of the Raf inhibitor immediately ${ }_{352}$ activated the ERK, and the ERK activity demonstrated slower 353 dynamics than that before the Raf inhibitor treatment and after ${ }_{354}$ the activation [37] (Fig. Tla and Supplementary Video 2). This 355 result implies that the thermodynamic cost of the ERK phos-356 phorylation is immediately increased when adding the Raf in-357 hibitor. After the dynamics of the relaxation on the upstream 358 pathways, the Raf inhibitor addition generally decreases the 359 thermodynamic cost of the ERK phosphorylation.
In Fig. Thb, we show the histograms of these informationgeometric quantities and the efficiency $\{\mathcal{C}, \mathcal{L}, \mathcal{V}, \eta\}_{d^{\prime}}^{p}$ before $\left(d^{\prime}=\right.$ pre $)$ and after $\left(d^{\prime}=\right.$ post $)$ the Raf inhibitor addition, where the subscript $d^{\prime} \in\{$ pre, post $\}$ regards the Raf inhibitor addition. The mean value of the action $\overline{\mathcal{C}_{d^{\prime}}^{p}}$ decreases when the Raf is inhibited $\overline{\mathcal{C}_{\text {pre }}^{p}}>\overline{\mathcal{C}_{\text {post }}^{p}}$, and the mean values differ by one order of magnitude (see also Extended Data Table 3). This result shows the Raf inhibitor addition reduces the thermodynamic cost of the ERK phosphorylation. The mean velocity and length also decrease when the Raf inhibitor is added, $\overline{\mathcal{V}_{\text {pre }}^{p}}>\overline{\mathcal{V}_{\text {post }}^{p}}$, and $\overline{\mathcal{L}_{\text {pre }}^{p}}>\overline{\mathcal{L}_{\text {post }}^{p}}$, while the transition time $t_{1}-t_{0}$ becomes longer by the Raf inhibitor addition (Extended Data Fig. 3 and see also Extended Data Table 3). Surprisingly, the mean value of the efficiency is robust to the Raf inhibitor addition $\overline{\eta_{\text {pre }}^{p}} \simeq \overline{\eta_{\text {post }}^{p}}$, while the peak of the histogram can be decreased after adding the Raf inhibitor. Moreover, the efficiency $\eta_{d^{\prime}}^{p}$ seems to have no correlation with $\mathcal{C}_{d^{\prime}}^{p}$ nor $\mathcal{V}_{d^{\prime}}^{p}$ (Extended Data Fig. 4 and see also Extended Data Table 4), and this robustness would not come from an artifact correlation between information-geometric quantities. The efficiency compensation of the ERK phosphorylation might exist when the upstream pathways are perturbed by the inhibitor.

In summary, we introduced information-geometric quantities as thermodynamic measures, and evaluated the speed limit's efficiency for the ERK phosphorylation dynamics in living cells at the single-cell level. Our method quantitatively clarifies the amount of information transferred in living systems based on information geometry and the conversion efficiency from the thermodynamic cost to the intrinsic speed, which complements other studies about measurements of informational quantities in biological systems such as the mutual information in signal transduction [46-48] and the Fisher information matrix on molecular networks [49, 50]. Our method is generally applicable to the activation process, and it widely exists in signaling pathways such as the G-protein coupled receptor pathways for sensation [II] and the receptor tyrosine kinase signaling pathways for cell proliferation [45]. For example, the state change from the active state $A_{\text {on }}$ to the inactive state $A_{\text {off }}$ in the G-protein coupled receptor corresponds to its conformational change by small rearrangements accompanying the ligand-binding. It is interesting to evaluate the speed limit's efficiency for other activation processes on an equal footing with this ERK phosphorylation in living NRK-52E cells. Our approach has great potential for other biological applications, which might clarify an efficient signal transduction mechanism and lead to novel insight into living systems as an information processing unit driven by the thermodynamic cost.
[1] Hopfield, J. J. Kinetic proofreading: a new mechanism forre-367 ducing errors in biosynthetic processes requiring high specificity.368 Proc. Natl. Acad. Sci. USA 71, 4135-4139 (1974).

[2] Ninio, J. Kinetic amplification of enzyme discrimination.370 Biochimie 57, 587-595 (1975).

[3] Lan, G., Sartori, P., Neumann, S., Sourjik, V. \& Tu, Y. The 372 energy-speed-accuracy trade-off in sensory adaptation. Nat. Phys. 8, 422-428 (2012).

[4] Mehta, P. \& Schwab, D. J. Energetic costs of cellular computation. Proc. Natl. Acad. Sci. USA 109, 17978-17982 (2012).

[5] Ito, S. \& Sagawa, T. Maxwell's demon in biochemical signal transduction with feedback loop. Nat. Commun. 6, 1-6 (2015). 

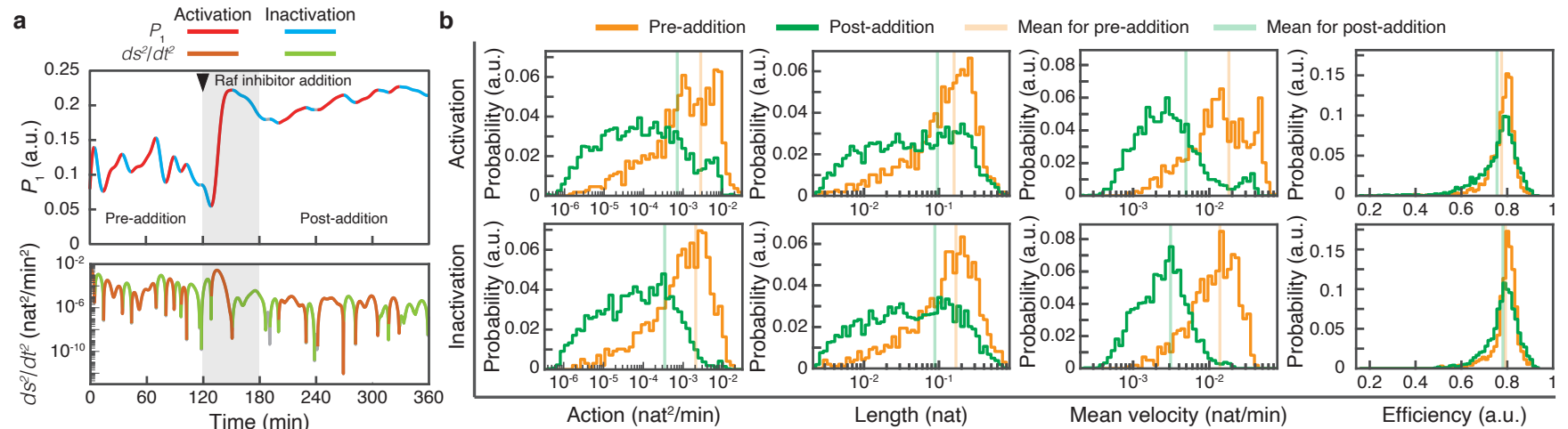

Fig. 4: The dynamics of the ERK phosphorylation under the Raf inhibitor addition. a Representative dynamics under the Raf inhibitor addition. The Raf inhibitor (100 $\mathrm{nM}$ of SB590885) was applied at $120 \mathrm{~min}$. We set the pre-addition region as the period from $0 \mathrm{~min}$ to $120 \mathrm{~min}$, and the post-addition region as the period after $180 \mathrm{~min}$. b The histograms of the action $\mathcal{C}$, length $\mathcal{L}$, mean velocity $\mathcal{V}$, and the efficiency $\eta$ before (pre-addition) and after (post-addition) adding the Raf inhibitor. The sample size, mean values, variances, and results of statistical tests are shown in Extended Data Table 1, 2.

[6] Parrondo, J. M., Horowitz, J. M. \& Sagawa, T. Thermodynamics 413 of information. Nat. Phys. 11, 131-139 (2015).

[7] Crooks, G. E. Measuring thermodynamic length. Phys. Rev. Lett.415 99, 100602 (2007).

[8] Ito, S. Stochastic thermodynamic interpretation of information 417 geometry. Phys. Rev. Lett. 121, 030605 (2018).

[9] Ciliberto, S. Experiments in stochastic thermodynamics: Short 419 history and perspectives. Phys. Rev. X 7, 021051 (2017).

[10] Collin, D. et al. Verification of the Crooks fluctuation theorem ${ }_{421}$ and recovery of RNA folding free energies. Nature 437, 231-234422 (2005).

[11] Hayashi, K., Ueno, H., Iino, R. \& Noji, H. Fluctuation theorem424 applied to $\mathrm{F}_{1}$-ATPase. Phys. Rev. Lett. 104, 218103 (2010). $\quad 425$

[12] Ito, S. \& Dechant, A. Stochastic time evolution, information 426 geometry, and the Cramér-Rao bound. Phys. Rev. X 10, 021056427 (2020).

[13] Yoshimura, K. \& Ito, S. Information geometric inequalities429 of chemical thermodynamics. Preprint at https://arxiv. ${ }_{430}$ ora/abs/2005.08444 (2020).

[14] Luttrell, L. M. Reviews in molecular biology and biotechnol-432 ogy: transmembrane signaling by $\mathrm{G}$ protein-coupled receptors. 433 Mol. Biotechnol. 39, 239-264 (2008).

[15] Seifert, U. Stochastic thermodynamics, fluctuation theorems 435 and molecular machines. Rep. Prog. Phys. 75, 126001 (2012). 436

[16] Rao, R. \& Esposito, M. Nonequilibrium thermodynamics of 437 chemical reaction networks: wisdom from stochastic thermody-438 namics. Phys. Rev. X 6, 041064 (2016).

[17] Ge, H. \& Qian, H. Nonequilibrium thermodynamic formalism 440 of nonlinear chemical reaction systems with Waage-Guldberg's441 law of mass action. Chem. Phys. 472, 241-248 (2016).

18] Ito, S. \& Sagawa, T. Information thermodynamics on causal 443 networks. Phys. Rev. Lett. 111, 180603 (2013).

[19] Barato, A. C., Hartich, D. \& Seifert, U. Efficiency of cellular 445 information processing. New J. Phys. 16, 103024 (2014). $\quad 446$

[20] Sartori, P., Granger, L., Lee, C. F. \& Horowitz, J. M. Thermo-447 dynamic costs of information processing in sensory adaptation.448 PLoS Comput. Biol. 10, e1003974 (2014).

[21] Barato, A. C. \& Seifert, U. Thermodynamic uncertainty relation 450 for biomolecular processes. Phys. Rev. Lett. 114, 158101 (2015).451

[22] Horowitz, J. M. \& Gingrich, T. R. Thermodynamic uncertainty 452 relations constrain non-equilibrium fluctuations. Nat. Phys. 16, 15-20 (2020)

[23] Shiraishi, N., Funo, K. \& Saito, K. Speed limit for classical stochastic processes. Phys. Rev. Lett. 121, 070601 (2018).

[24] Ashida, K. \& Oka, K. Stochastic thermodynamic limit on E. coli adaptation by information geometric approach. Biochem. Biophys. Res. Commun. 508, 690-694 (2019).

[25] Dechant, A. \& Sakurai, Y. Thermodynamic interpretation of Wasserstein distance. Preprint at https://arxiv.org/ abs / 1912.08405 (2019).

[26] Gupta, D. \& Busiello, D. M. Tighter thermodynamic bound on speed limit in systems with unidirectional transitions. Preprint at https://arxiv.org/abs/2009.11115 (2020).

[27] Zhang, Z., Guan, S. \& Shi, H. Information geometry in the population dynamics of bacteria. J. Stat. Mech. 2020, 073501 (2020).

[28] Falasco, G. \& Esposito, M. Dissipation-time uncertainty relation. Phys. Rev. Lett. 125, 120604 (2020).

[29] Nicholson, S. B., Garcia-Pintos, L. P., del Campo, A. \& Green, J. R. Time-information uncertainty relations in thermodynamics. Nat. Phys. (2020).

[30] Dechant, A. Multidimensional thermodynamic uncertainty relations. J. Phys. A 52, 035001 (2018).

[31] Amari, S. Information Geometry and Its Applications. (Springer, Japan, 2016).

[32] Ruppeiner, G. Riemannian geometry in thermodynamic fluctuation theory. Rev. Mod. Phys. 67, 605 (1995).

[33] Ito, S., Oizumi, M. \& Amari, S. Unified framework for the entropy production and the stochastic interaction based on information geometry. Phys. Rev. Research 2, 033048 (2020).

[34] Pietzonka, P., Ritort, F. \& Seifert, U. Finite-time generalization of the thermodynamic uncertainty relation. Phys. Rev. E 96, 012101 (2017).

[35] Paneru, G., Dutta, S., Tlusty, T. \& Pak, H. K. Reaching and violating thermodynamic uncertainty bounds in information engines. Phys. Rev. E 102, 032126 (2020).

[36] Albeck, J. G., Mills, G. B. \& Brugge, J. S. Frequencymodulated pulses of ERK activity transmit quantitative proliferation signals. Mol. Cell 49, 249-261 (2013).

[37] Aoki, K. et al. Stochastic ERK activation induced by noise and cell-to-cell propagation regulates cell density-dependent prolif- 
eration. Mol. Cell 52, 529-540 (2013).

[38] Yang, J. M. et al. Integrating chemical and mechanical signals through dynamic coupling between cellular protrusions and pulsed ERK activation. Nat. Commun. 9, 4673 (2018).

[39] Lavoie, H., Gagnon, J. \& Therrien, M. ERK signalling: a master regulator of cell behaviour, life and fate. Nat. Rev. Mol. Cell Biol. 21, 607-632 (2020).

[40] Knowles, J. R. Enzyme-catalyzed phosphoryl transfer reactions. Annu. Rev. Biochem. 49, 877-919 (1980).

[41] Komatsu, N. et al. Development of an optimized backbone of FRET biosensors for kinases and GTPases. Mol. Biol. Cell 22, 4647-4656 (2011).

[42] Hatzivassiliou, G. et al. RAF inhibitors prime wild-type RAF to activate the MAPK pathway and enhance growth. Nature $\mathbf{4 6 4}$, 431-435 (2010).

[43] Heidorn, S. J. et al. Kinase-dead BRAF and oncogenic RAS cooperate to drive tumor progression through CRAF. Cell 140, 209-221 (2010).

[44] Poulikakos, P. I. et al. RAF inhibitor resistance is mediated by dimerization of aberrantly spliced BRAF (V600E). Nature 480, 387-390 (2011).

[45] Lemmon, M. A. \& Schlessinger, J. Cell signaling by receptor tyrosine kinases. Cell 141, 1117-1134 (2010).

[46] Mehta, P., Goyal, S., Long, T., Bassler, B. L. \& Wingreen, N. S. Information processing and signal integration in bacterial quorum sensing. Mol. Syst. Biol. 5, 325 (2009).

[47] Uda, S. et al. Robustness and compensation of information transmission of signaling pathways. Science 341, 558-561 (2013).

[48] Selimkhanov, J. et al. Accurate information transmission through dynamic biochemical signaling networks. Science 346, 1370-1373 (2014).

[49] Machta, B. B., Chachra, R., Transtrum, M. K. \& Sethna, J. P. Parameter space compression underlies emergent theories and predictive models. Science 342, 604-607 (2013).

[50] Singh, V. \& Nemenman, I. Universal properties of concentration sensing in large ligand-receptor networks. Phys. Rev. Lett. 124, 028101 (2020). 
We measured the ERK phosphorylation, as previously de- ${ }^{547}$ scribed [5]]. The EKAREV-NLS stable-expression NRK-52E cell lines (NRK-52E/ERKAREV-NLS cells) [37], were used, and the EKAREV-NLS is the genetically encoded ERK sensor ${ }_{551}$ used in Komatsu et al., 2011 [41]].

The NRK-52E/EKAREV-NLS cells were maintained with $_{554}$ the Dulbecco's Modified Eagle Medium (DMEM; Ther-555 moFisher), $10 \%$ Fetal bovine serum (FBS; Sigma), and $10_{556}$ $\mu \mathrm{g} / \mathrm{mL}$ Blasticidin $\mathrm{S}$ (Invitrogen). The cells were seeded at $\mathrm{a}_{557}$ specific concentration (Low: $2.0 \times 10^{3}$ cells $/ \mathrm{cm}^{2}$, Medium:558 $2.0 \times 10^{4} \mathrm{cells} / \mathrm{cm}^{2}$, High: $\left.2.0 \times 10^{5} \mathrm{cells} / \mathrm{cm}^{2}\right)$ on glass-559 bottom dishes (IWAKI). One day after the seeding, the ${ }_{560}$ time-lapse imaging was performed. The culture media was $_{561}$ replaced with the FluoroBrite (ThermoFisher), $5 \% \mathrm{FBS}_{562}$ (Sigma), and $1 \times$ Glutamax (ThermoFisher) 3-6 hours before ${ }_{563}$ starting the time-lapse imaging. We used an inverted micro-564 scope (IX81; Olympus) equipped with a CCD camera (Cool-565 SNAP K4; Roper Scientific) and an excitation light source 566 (Spectra-X light engine; Lumenncor). Optical filters were ${ }_{567}$ as follows: an FF01-438/24 excitation filter (Semrock), an ${ }_{568}$ XF2034 (455DRLP) dichroic mirror (Omega Optical), and $\mathrm{d}_{569}$ two emission filters (FF01-483/32 for CFP and FF01-542/27 for YFP (Semrock)). Images were acquired every $20 \mathrm{sec}$ (the ${ }_{571}$ exposure time was $100 \mathrm{~ms}$ ) with binning $8 \times 8$ on MetaMorph H72 $_{72}$ software (Universal Imaging) with an IX2-ZDC laser-based ${ }_{573}$ autofocusing system (Olympus). A $\times 20$ lens $\left(\mathrm{UPLSAPO}_{574}\right.$ 20X; Olympus, numerical aperture: 0.75 ) was used. The tem-575 perature and $\mathrm{CO}_{2}$ concentration were maintained at $37^{\circ} \mathrm{C}_{\text {and }}{ }_{576}$ $5 \%$ during the imaging with a stage top incubator (Tokai ${ }_{577}$ hit). For the Raf inhibitor experiment, the experiment was $_{578}$ performed under the same condition as the medium cell den-579 sity condition. We applied SB590885 (Selleck Chemicals) $\left(\mathrm{a}_{580}\right.$ final concentration is $100 \mathrm{nM}$ ) 2 hours after the imaging initi- $-_{581}$ ation. The numbers of trials for each experimental condition $_{582}$ are two.

We used the same relation between the FRET/CFP ratios of the EKAREV-NLS and the phosphorylated ERK fraction (pTpY-ERK2) from the western blotting in Fig. $2 \mathbf{b}$ as de-585 scribed previously [37]. In brief, the phosphorylated ERK fraction was quantified by the Phos-tag western blotting [52] in HeLa cells stimulated with different concentrations of $12^{586}$ O-Tetradecanoylphorbol 13-acetate (TPA; Sigma) for $30 \mathrm{~min}^{587}$ to induce the ERK phosphorylation. Under the same condi- ${ }^{588}$ tion, HeLa cells stably expressing EKAREV-NLS were imaged, followed by the quantification of the average FRET/CFP ratios. Finally, the FRET/CFP ratios were plotted as a func- ${ }^{589}$ tion of the phosphorylated ERK fraction with the fitted curve of the Hill equation shown in Fig. Zb by the Solver Add-in ins9o Excel (Microsoft).
For the imaging analysis, Fiji was used. The background was subtracted by the Subtract Background Tool, and after that, the nuclei were tracked with a custom-made tracking program. Only the cells which were tracked over the entire images were used for calculation of information-geometric quantities. The phosphorylated ERK fraction was calculated based on the FRET/CFP ratios with the Hill equation shown in Fig. Zlb.

For analysis of the time-series phosphorylated ERK fractions, MATLAB 2019b (MathWorks) was used. The low-pass filter, whose cutoff frequency is $0.005 \mathrm{~Hz}$, was used for the cell density experiment data with the designfilt function in the Signal Processing Toolbox to reduce the noise. The low-pass filter, whose cutoff frequency is $1 / 1200 \mathrm{~Hz}$, was used for the Raf inhibitor addition experiment data to detect the slower dynamics after the Raf inhibitor addition. We identified the activation and inactivation processes from the signs of the first and second derivatives. The first derivatives were numerically calculated using two data points of the phosphorylated fraction, and the second derivatives were numerically calculated using two data points of the first derivatives. We only calculated information-geometric quantities when the phosphorylated ERK fractions between maximum and minimum of the process over 0.01 for the density-experiment data and 0.001 for the Raf inhibitor addition experiment data. The square of an intrinsic speed $d s^{2} / d t^{2}$ was numerically calculated using two-points of the phosphorylated ERK fraction time series. The action $\mathcal{C}$, length $\mathcal{L}$, mean velocity $\mathcal{V}$, and efficiency $\eta$ were calculated using $d s^{2} / d t^{2}$ according to the definitions. We used the set of time series for each cell in two trials for each experimental condition to make histograms.

For the statistical analysis, $\mathrm{R}$ (version 3.6.3; R project) was used. The Brunner-Munzel test was performed with the brunnermunzel.test function in the brunnermunzel library (version 1.4.1). The two-sample Kolmogorov-Smirnov test was performed with the ks.test function in the stats library (version 3.6.3). The Pearson correlation coefficients were calculated and tested by the cor.test function in the stats library (version 3.6.3). The Holm method was used to control the family-wise error rates with the p.adjust function in the stats library (version 3.6.3). The sample numbers are shown in Extended Data Table 1.

\section{DATA AVAILABILITY}

The data sets generated and analyzed during the current study are available from the corresponding author on reasonable request.

\section{CODE AVAILABILITY}

The source codes used in the current study are available from the corresponding author on reasonable request. 
experiments. Keita Ashida analyzed the data. All authors discussed the analytical results of the experiment and wrote the paper.
S. I. is supported by JSPS KAKENHI Grants No. ${ }^{608}$

S. I. proposed the main method based on information geometry and designed the research. Kazuhiro Aoki performed 610

JP19H05796, and JST Presto Grant No. JP18070368, Japan.

Kazuhiro Aoki is supported by JSPS KAKENHI Grants No.

JP19H05798. S. I. and Keita Ashida thank Kohei Yoshimura

for discussions of chemical thermodynamics, S. I thanks Keita

Kamino for the discussion of experimental difficulties in measurement of information quantity of living cells, and Kazuhiro Aoki thanks Yohei Kondo for helpful discussions of thermodynamics. S. I. also thanks Kiyoshi Kanazawa for careful reading of this manuscript.

\section{AUTHOR CONTRIBUTIONS}

1 [51] Aoki, K. \& Matsuda, M. Visualization of small GTPase activity with fluorescence resonance energy transfer-based biosensors. Nat. Protoc. 4, 1623-1631 (2009).

[52] Kinoshita, E., Kinoshita-Kikuta, E., Takiyama, K. \& Koike, T. Phosphate-binding tag, a new Tool to visualize phosphorylated proteins. Mol. Cell. Proteom. 5, 749-757 (2006). 


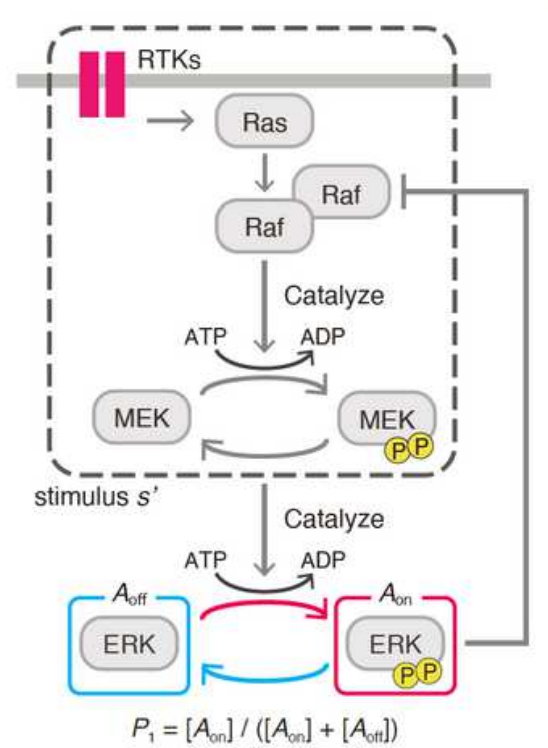

b

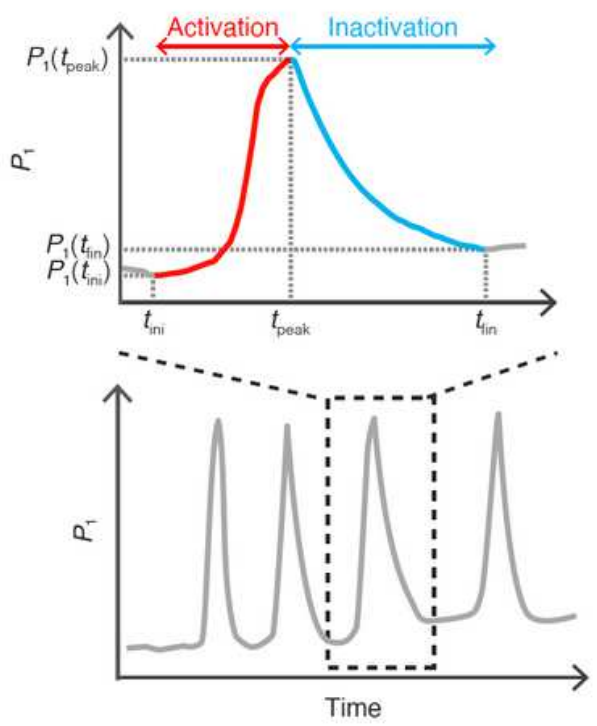

c
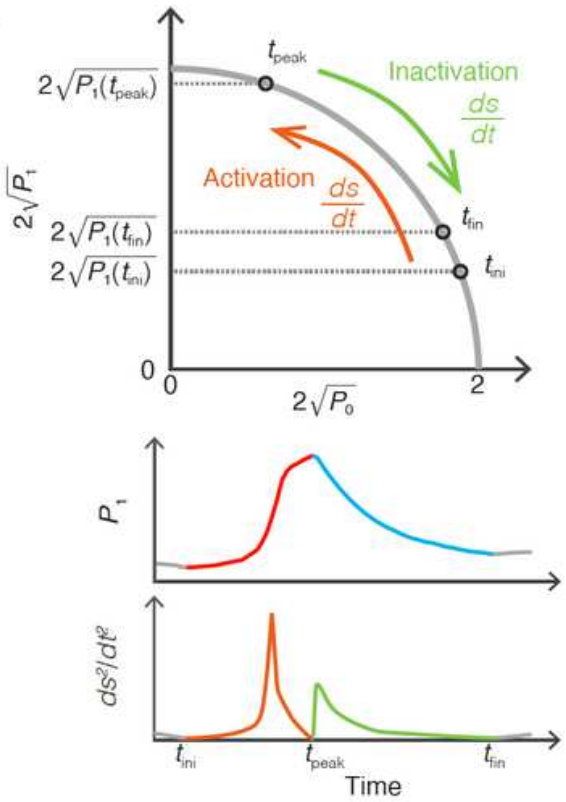

Figure 1

Schematic of the ERK phosphorylation, the time series of the phosphorylated ERK fraction P1, and the square of the intrinsic speed ds2/dt2. a The Raf-MEK-ERK MAP pathway as an example of switching dynamics between the active state Aon and the inactive state Aoff . The phosphorylated and nonphosphorylated ERKs correspond to Aon and Aoff, respectively. The upstream proteins correspond to the stimulus s', and the Raf on the upstream pathway affects the phosphorylated ERK. b Typical behavior of the phosphorylated ERK fraction P1 in the activation and inactivation processes. The time tini (tpeak) indicates the beginning of the activation (inactivation), and the time tpeak (tfin) indicates the end of the activation (inactivation). c Schematic of the manifold in information geometry and the intrinsic speed $\mathrm{ds} / \mathrm{dt}$ on this manifold. The activation and inactivation processes give at least two peaks in the time series of $\mathrm{ds} 2 / \mathrm{dt} 2$, because $\mathrm{ds} 2 / \mathrm{dt} 2=0$ at times tini, tpeak, and tfin.

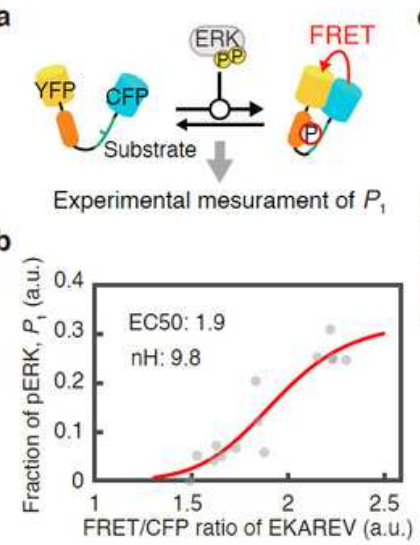

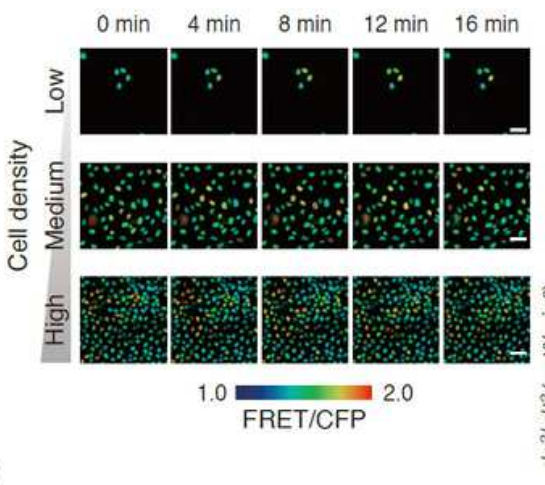
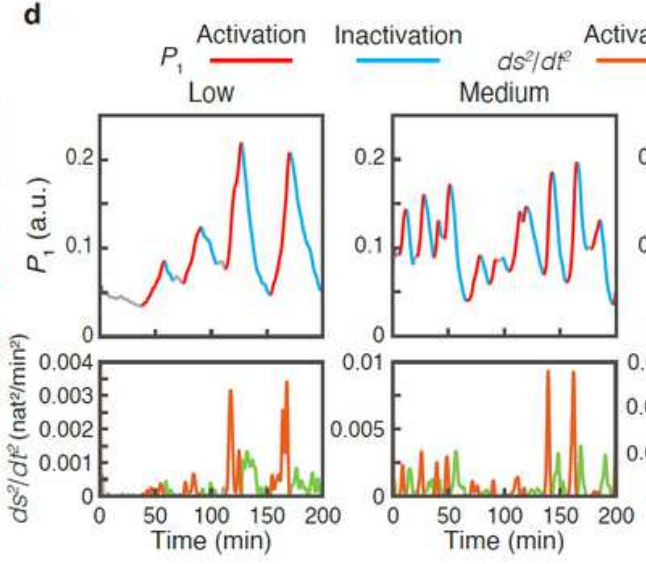

Activation Inactivation

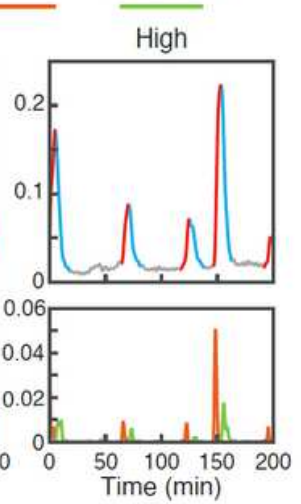

Figure 2 
The dynamics of the ERK phosphorylation under the conditions of different cell densities. a Schematic of the FRET-based ERK sensor. The phosphorylated ERK fraction P1 can be measured from this FRET signal. $b$ The relation between the FRET/CFP ratios of the EKAREV-NLS and the phosphorylated ERK fraction P1. The red line indicates the fitted curve of the Hill equation. c Representative images of the phosphorylated ERK under the conditions of low, medium, and high cell densities. Scale bars indicate $50 \mu \mathrm{m}$. $\mathrm{d}$ Representative examples of the ERK phosphorylation dynamics under low (left), medium (middle), and high (right) cell densities. The unit (a.u.) indicates the arbitrary unit, and the unit (nat) indicates the natural unit of information.

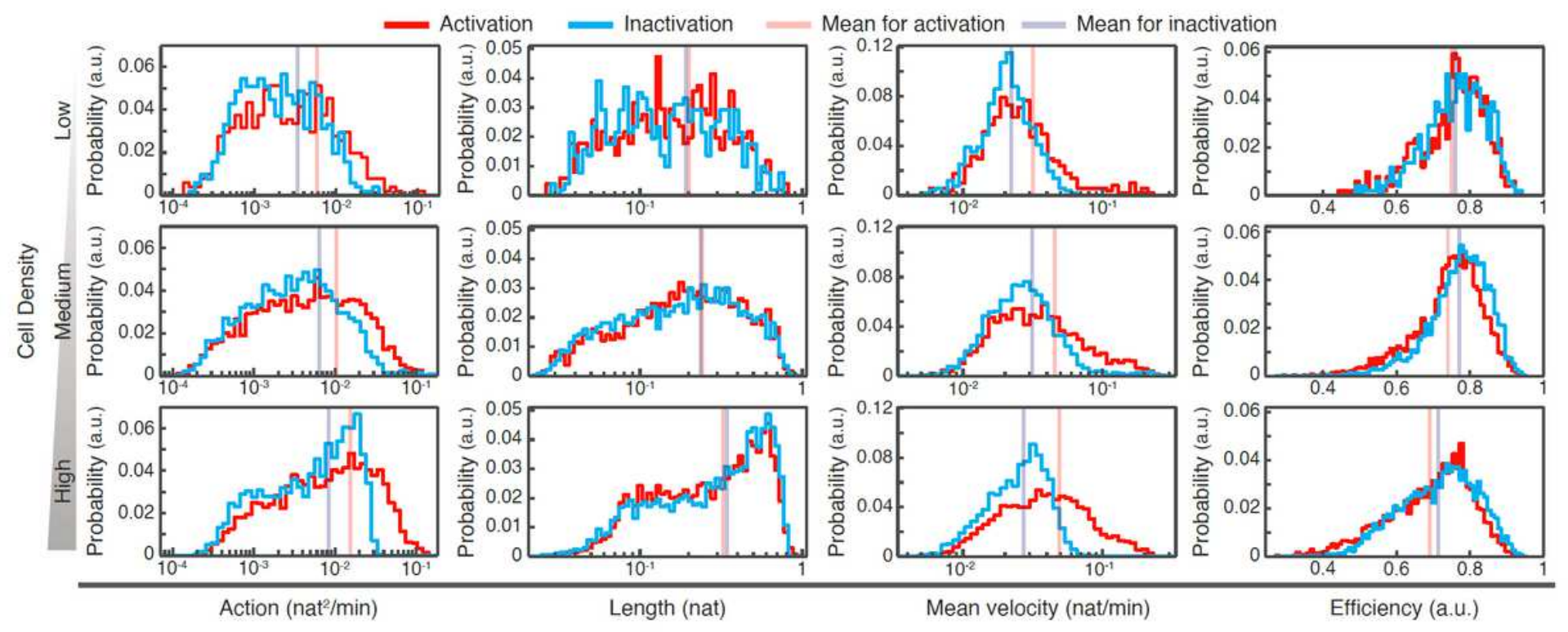

\section{Figure 3}

The histograms of the action $C$, length $L$, mean velocity $V$, and efficiency $\eta$ under the conditions of different cell densities. The sample size, mean values, variances, and results of statistical tests are listed in Extended Data Table 1, 2.
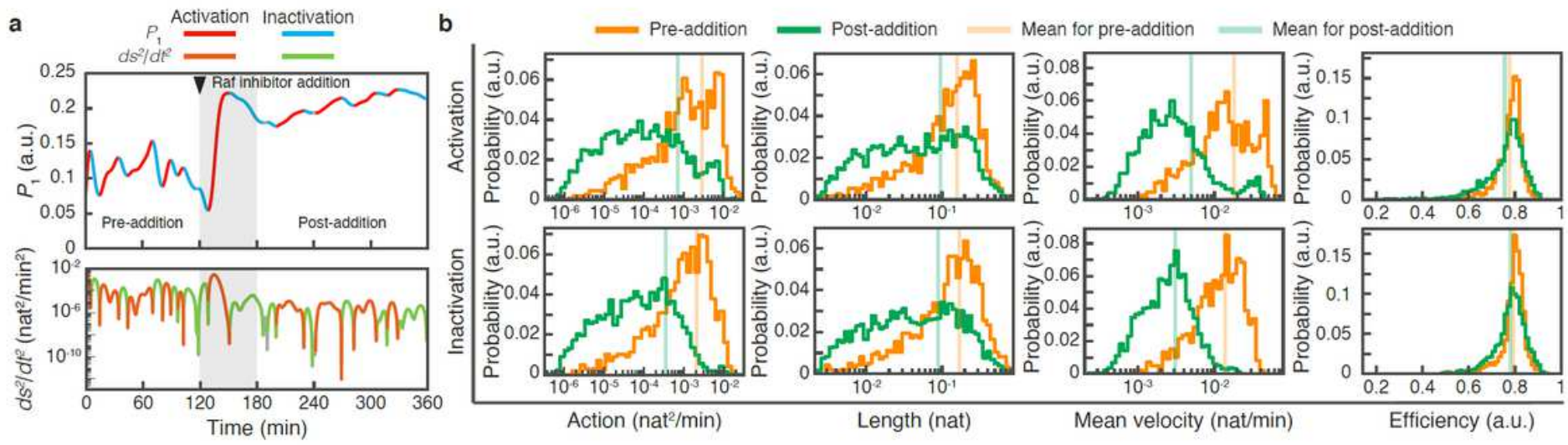

Figure 4 
The dynamics of the ERK phosphorylation under the Raf inhibitor addition. a Representative dynamics under the Raf inhibitor addition. The Raf inhibitor (100 nM of SB590885) was applied at $120 \mathrm{~min}$. We set the pre-addition region as the period from $0 \mathrm{~min}$ to $120 \mathrm{~min}$, and the post-addition region as the period after 180 min. b The histograms of the action $C$, length $L$, mean velocity $V$, and the efficiency $\eta$ before (pre-addition) and after (post-addition) adding the Raf inhibitor. The sample size, mean values, variances, and results of statistical tests are shown in Extended Data Table 1, 2.

\section{Supplementary Files}

This is a list of supplementary files associated with this preprint. Click to download.

- VideoS1.mov

- Videos2.mov

- supplements.pdf 